Submitted to

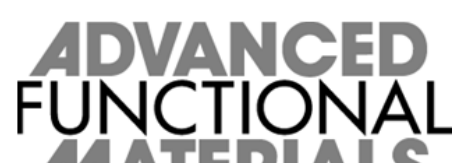

DOI: $10.1002 /$ adfm.201302173

\title{
Influence of cavity thickness and emitter orientation on the efficiency roll-off of phosphorescent organic light-emitting diodes
}

By Caroline Murawski ${ }^{\dagger}$, Philipp Liehm ${ }^{\dagger}$, Karl Leo, and Malte C. Gather*\#

[*] C. Murawski, P. Liehm, Prof. K. Leo, Jun.-Prof. M. C. Gather

Institut für Angewandte Photophysik

Technische Universität Dresden

George-Bähr-Str. 1, 01062 Dresden (Germany)

E-mail: caroline.murawski@iapp.de, malte.gather@iapp.de

$\left.{ }^{\#}\right] \quad$ Present address: Prof. M. C. Gather

SUPA, School of Physics and Astronomy

University of St Andrews

St Andrews KY16 9SS (UK)

$\left[{ }^{\dagger}\right] \quad$ These authors contributed equally to this work.

Keywords: OLED, efficiency roll-off, emitter orientation, decay rate, triplet-tripletannihilation

\section{Abstract}

This article describes the first systematic investigation of how the efficiency roll-off in organic light-emitting diodes (OLEDs) is influenced by the position and orientation of the emitter molecules within the OLED cavity. The efficiency roll-off is investigated for two OLED stacks containing either the phosphorescent emitter $\operatorname{Ir}(\mathrm{MDQ})_{2}(\mathrm{acac})$ or $\operatorname{Ir}(\mathrm{ppy})_{3}$ by varying the distance between emitter and metal cathode; a strong influence of emitter position and orientation on roll-off is observed. The measurements are modeled by triplet-tripletannihilation (TTA) theory yielding the critical current density and the TTA rate constant. We find that $\operatorname{Ir}(\mathrm{MDQ})_{2}(\mathrm{acac})$ shows the lowest roll-off when the emitter is located in the first optical maximum of the electromagnetic field, whereas the roll-off of the $\operatorname{Ir}(\text { ppy })_{3}$ stack is lowest when the emitter is positioned closer to the metal cathode. Measurement and modeling of time-resolved electroluminescence show that the different roll-off behavior is due to the 


\section{Submitted to

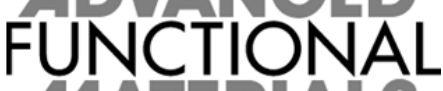

different orientation and the corresponding change of the decay rate of the emissive dipoles of $\operatorname{Ir}(\mathrm{MDQ})_{2}(\mathrm{acac})$ and $\operatorname{Ir}(\mathrm{ppy})_{3}$. Finally, we develop design principles for optimal highbrightness performance by modeling the roll-off as a function of emitter-cathode distance, emissive dipole orientation, and radiative efficiency.

\section{Introduction}

Organic light-emitting diodes (OLEDs) are a novel type of light source that provides homogeneous and glare-free illumination over an extended area using thin films of luminescent organic molecules. In conventional OLEDs, the emitter molecules are fluorescent, i.e., they only emit from their singlet state and thus typically reach internal quantum efficiencies below 25\%. ${ }^{[1]}$ By instead using phosphorescent materials, which emit from the triplet state, internal quantum efficiencies close to $100 \%$ can be reached. Based on this concept, devices efficiencies that are comparable with fluorescent tubes have already been demonstrated, at least at moderate brightness levels. ${ }^{[2,3]}$

However, the efficiency of OLEDs typically decreases when operated at high brightness, an effect known as efficiency roll-off. As future applications of the OLED technology in general illumination will require operation at high brightness levels, understanding and reducing this roll-off is of major importance. The efficiency roll-off results from quenching of excitons by other excitons, polarons, or the electric field, or can be due to a change in charge carrier balance. ${ }^{[4-7]}$ Among these mechanisms, triplet-triplet-annihilation (TTA) was found to be particularly important for devices based on phosphorescent emitter molecules. ${ }^{[5,7]}$ In the TTA process, the annihilation of two triplet excitons, $T_{1}$, can be described as

$$
T_{1}+T_{1} \stackrel{k_{\mathrm{TT}}}{\rightarrow} T_{\mathrm{n}}+S_{0} \rightarrow T_{1}+S_{0}
$$




\section{Submitted to

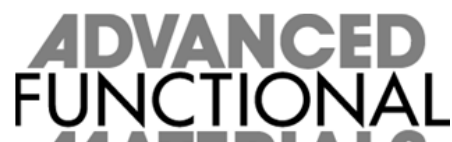 \\ MATERIALS}

where $k_{\mathrm{TT}}$ is the TTA rate constant and $T_{\mathrm{n}}$ and $S_{0}$ refer to a higher excited triplet state and the singlet ground state of the molecules, respectively. The TTA process thus yields one excited triplet and one ground state molecule. The number of TTA events depends not only on $k_{\mathrm{TT}}$, but also on the triplet density within the emissive region of the device and on the excited state lifetime of the emitter (triplet lifetime). Strategies to reduce the roll-off and thus increase the high-brightness performance of OLEDs include reducing the triplet density and/or the triplet lifetime of the emitter molecules. The triplet density correlates with the width of the triplet exciton formation zone, i.e., the region of the device in which excitons are created by recombination of electrons and holes, and mainly depends on the charge transport characteristics of the emission layer (EML). Very broad exciton formation zones have recently been demonstrated using graded EML structures, where a preferentially electron transporting matrix material is doped into a hole transporting material at a concentration that changes continuously across the thickness of the EML. ${ }^{[8]}$

The other critical parameter, the triplet lifetime, is in the range of $1 \mu \mathrm{s}$ for state-of-theart phosphorescent emitters. In these emitters, the introduction of heavy metal atoms such as iridium allows efficient intersystem crossing from the singlet to the triplet state and thus enables high rates of radiative phosphorescence. ${ }^{[9-11]}$ However, even for these phosphorescent emitters the triplet lifetime is still approximately three orders of magnitude higher than the fluorescence decay time of conventional singlet emitters, which results in a rather high efficiency roll-off of phosphorescent OLEDs. To further decrease the triplet lifetime, several strategies were proposed: For instance, changing the molecular design by using active hydrogen in a phosphorescent Ir-complex resulted in a very low triplet lifetime of only $3.7 \mathrm{~ns}^{[12]}$ 


\section{Submitted to}

Another strategy is to modify the triplet lifetime by changing the optical environment of the emissive molecule. ${ }^{[13]}$ By placing the emitter within a strong microcavity or in close proximity to metal surfaces, one can considerably reduce the effective triplet lifetime. While the intrinsic triplet lifetime is a material parameter and denotes the lifetime the emitter molecule would have in free space, the effective lifetime takes the influence of the optical environment into account. ${ }^{[14-16]}$ For instance, improved roll-off has been demonstrated for top-emitting OLEDs, where the presence of a stronger microcavity than in conventional bottom-emitting structures shortens the emitter lifetime. ${ }^{[17]}$ Song et al. have found some evidence that the roll-off also correlates with the distance between the emitter and the reflecting metal cathode. ${ }^{[18]}$ However, the effect has not been analyzed quantitatively and we suspect that in the study by Song et al. additional quenching mechanisms overlapped with TTA because the efficiency roll-off observed for large distances between the emitter and the cathode was higher than expected under the TTA model.

In this article, we systematically vary the distance of the emitter molecules from the reflecting metal cathode in OLEDs based on two different Ir-complexes in order to investigate the influence of the optical environment on the efficiency roll-off. We compare our results to direct measurements and optical simulations of the effective triplet lifetime. In contrast to previous reports, ${ }^{[18]}$ which claimed that TTA is generally reduced by close proximity of the emitter molecules to a metal electrode, our analysis reveals that the TTA behavior and, thus, the efficiency roll-off depend on emitter orientation. The TTA rate constant $k_{\mathrm{TT}}$ of the different emitter materials is determined by combining the measurements of efficiency roll-off with time-resolved measurements. Finally, we model the efficiency roll-off as a function of the emitter-cathode distance for different emitter orientations and different intrinsic radiative efficiencies to establish design principles for OLEDs with optimized roll-off characteristics. 
Submitted to

\section{Results and Discussion}

Schematics of the OLED layer stacks investigated in this study are shown in Figure 1a and b. Stack A contains the red-emitting $\operatorname{Ir}(\mathrm{MDQ})_{2}(\mathrm{acac})$ in an NPB matrix while Stack B is based on the green-emitting $\operatorname{Ir}(\mathrm{ppy})_{3}$ in a double-EML consisting of the hole-transporting TCTA and the electron-transporting TPBi. The distance of the EML to the metal cathode is varied by changing the thickness of the electron transport layer (ETL) over a broad range (Stack A from 25 to $265 \mathrm{~nm}$ and Stack B from 30 to $255 \mathrm{~nm}$ ). We employ electrically doped transport layers to ensure that all samples have similar electrical performance despite their significantly different overall thickness. ${ }^{[19]}$ In fact, up to a current density of $3 \mathrm{~mA} \mathrm{~cm}{ }^{-2}$, identical current-voltage characteristics are observed for all ETL thicknesses (cf. Figure 1c). At higher current densities, a slight decrease of the current density with increasing ETL thickness is observed.

\subsection{Dependence of efficiency roll-off on emitter-cathode distance}

Figure 2a shows the measured external quantum efficiency (EQE) for all samples as a function of the ETL thickness. The EQE values were taken at a current density of $0.5 \mathrm{~mA} \mathrm{~cm}$ ${ }^{2}$, where TTA rates are negligible. The outcoupling efficiency varies depending on the position of the emitter with respect to the electromagnetic field supported by the OLED stack, which leads to pronounced EQE maxima and minima for certain ETL thickness. ${ }^{[20]}$ The devices at the two EQE maxima are referred to in the following as first maximum device (for the lower ETL thickness) and second maximum device (for the thicker ETL). The highest EQE values are achieved at an ETL thickness of $265 \mathrm{~nm}$ for $\operatorname{Ir}(\mathrm{MDQ})_{2}$ (acac) and at $205 \mathrm{~nm}$ for $\operatorname{Ir}(\mathrm{ppy})_{3}$, which corresponds to the second maximum in both cases. The fact that the EQE 


\section{Submitted to

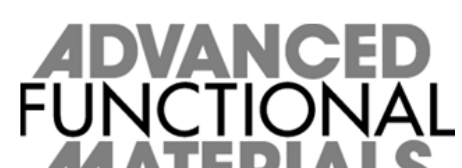

of the second maximum device is higher than of the first maximum device is a result of the high radiative efficiency of $\operatorname{Ir}(\mathrm{MDQ})_{2}(\mathrm{acac})$ and $\operatorname{Ir}(\mathrm{ppy})_{3} \cdot{ }^{[20]}$

Figure $2 \mathrm{~b}$ shows the EQE as a function of the current density $J$ for four $\operatorname{Ir}(\mathrm{MDQ})_{2}(\mathrm{acac})$ devices with different ETL thickness. All four datasets are normalized to the efficiency at low current density where TTA is negligible. The roll-off (i.e., the relative decrease in EQE at high current densities) indeed shows a pronounced variation between devices, but no direct proportionality between roll-off and ETL thickness can be seen, contrary to previous reports. ${ }^{[18]}$ A good measure for the roll-off is the critical current density $J_{0}$, i.e., the current density at which the EQE has decreased to half of its initial value. ${ }^{[4]}$ However, a direct measurement of $J_{0}$ is usually not possible or not meaningful because very high current densities would have to be applied, which would lead to device degradation. ${ }^{[21]}$ (In this context, we note that the roll-off shown in Figure $2 b$ was reversible, i.e., the original EQE was fully recovered when going back to low current densities.) We therefore fitted the normalized experimental EQE vs. $J$ data with the expected behavior and extrapolated the fit up to the critical current density as described in the following: Under electrical excitation, the triplet exciton density $n(t)$ in OLEDs is described by the following rate equation, assuming TTA is the only relevant quenching process: $:^{[4,5]}$

$$
\frac{d}{d t} n(t)=-\frac{n(t)}{\tau^{*}}-\frac{1}{2} k_{\mathrm{TT}} n(t)^{2}+\frac{J}{q w} .
$$

Here, $\tau^{*}$ is the effective emitter lifetime, $q$ is the elementary charge, and $w$ is the width of the exciton formation zone. Solving this equation yields the normalized EQE

$$
\frac{\mathrm{EQE}}{\mathrm{EQE}_{0}}=\frac{J_{0}}{4 J}\left(\sqrt{1+8 \frac{J}{J_{0}}}-1\right), \text { with }
$$




$$
J_{0}=\frac{4 q w}{k_{\mathrm{TT}} \tau^{* 2}}
$$

Here, $\mathrm{EQE}_{0}$ is the $\mathrm{EQE}$ at low current density, where no TTA occurs. According to Eq. (4), the critical current density $J_{0}$ depends on the width of the exciton formation zone $w$, the TTA rate constant $k_{\mathrm{TT}}$, and the effective triplet lifetime $\tau^{*}$. Here, the triplet lifetime has the strongest influence due to the $1 / \tau^{* 2}$ dependence.

Next, the measured EQE data are fitted by Eq. (3), yielding $J_{0}$ as a measure of roll-off. Figure 3 summarizes the extracted $J_{0}$ values for both samples as a function of ETL thickness. For the $\operatorname{Ir}(\mathrm{MDQ})_{2}(\mathrm{acac})$ based device, the highest critical current density and, thus, the lowest roll-off is observed for ETL thicknesses close to the first EQE maximum (cf. Figure 2a), i.e., for ETL thicknesses of $50-100 \mathrm{~nm}$. The lowest $J_{0}$ value is obtained at $160 \mathrm{~nm}$, close to the optical minimum. The behavior is different for the $\operatorname{Ir}(\mathrm{ppy})_{3}$ based devices: Here, the highest $J_{0}$ (lowest roll-off) is obtained when the emitter molecules are in close proximity to the metal cathode (thin ETL), while $J_{0}$ stays relatively constant for ETL thicknesses above $100 \mathrm{~nm}$. For both emitters, critical current densities between 150 and $350 \mathrm{~mA} \mathrm{~cm}^{-2}$ are achieved, which are typical values for state-of-the-art OLEDs. ${ }^{[7]}$

\subsection{Optical simulation of the emitter lifetime}

To establish why the $\operatorname{Ir}(\mathrm{MDQ})_{2}(\mathrm{acac})$ based OLEDs behave differently than the $\operatorname{Ir}(\mathrm{ppy})_{3}$ devices, we consider the effective triplet decay rate $\Gamma^{*}$ of the emitters, which is the inverse of the effective triplet lifetime $\tau^{*}$. An excited emitter molecule either decays radiatively into a photon or into non-radiative modes, e.g., phonons. $\Gamma^{*}$ represents the sum of the effective radiative decay rate $\Gamma_{\mathrm{r}}^{*}=F \Gamma_{\mathrm{r}}$ and the intrinsic non-radiative decay rate $\Gamma_{\mathrm{nr}}$ : 


$$
\Gamma^{*}=\frac{1}{\tau^{*}}=F \Gamma_{\mathrm{r}}+\Gamma_{\mathrm{nr}}
$$

Here, $F$ is the Purcell factor, i.e., the ratio between the total power radiated by the molecule within a specific optical environment (e.g., close to the metal contact of an OLED) and the power the molecule would emit in free space. We calculate $F$ for the given layer structure by approximating the emitter molecules as oscillating dipoles and using a transfer-matrixapproach. ${ }^{[20]}$ As $F$ depends on the orientation of the dipole, the average $F$ for an ensemble of dipoles is given by the weighted average of the Purcell factors for the three principal transition dipole orientations of the emitter molecules. The orientations are typically distinguished by their polarization (transverse magnetic (TM) or transverse electric (TE)) and by their orientation with respect to the plane of the device (horizontal (h) or vertical (v)):

$$
F=a F_{\mathrm{TM}, \mathrm{v}}+(1-a) \times\left(F_{\mathrm{TM}, \mathrm{h}}+F_{\mathrm{TE}, \mathrm{h}}\right) .
$$

To reflect the average orientation of the transition dipoles, the vertical and horizontal components are weighted by the anisotropy factor, $a$. For an isotropic orientation of transition dipoles $a=1 / 3$; $a$ takes values smaller (larger) than $1 / 3$ if the dipoles are mainly horizontally (vertically) oriented.

Finally, the intrinsic radiative efficiency $\eta_{\mathrm{r}}$ describes how efficient an excited molecule relaxes into a photon:

$$
\eta_{\mathrm{r}}=\frac{\Gamma_{\mathrm{r}}}{\Gamma_{\mathrm{r}}+\Gamma_{\mathrm{nr}}} .
$$

To illustrate the influence of $F, a$, and $\eta_{\mathrm{r}}$ on $\Gamma^{*}$, Figure 4 shows the normalized decay rate

$$
\frac{\Gamma^{*}}{\Gamma}=\frac{\Gamma_{\mathrm{r}}^{*}+\Gamma_{\mathrm{nr}}}{\Gamma_{\mathrm{r}}+\Gamma_{\mathrm{nr}}}
$$




\section{Submitted to}

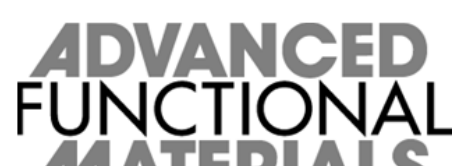

as a function of the ETL thickness for (a) different $a$ at $\eta_{\mathrm{r}}=0.8$ and (b) different $\eta_{\mathrm{r}}$ at $a=1 / 3$, assuming the layer structure given in Figure 1a. Variations of the dipole orientation $a$ show a pronounced influence on the evolution of the normalized decay rate, especially for small ETL thicknesses. This is a result of the strong coupling between vertically oriented dipoles and surface plasmon polaritons at the interface between the organic layers and the metal cathode. ${ }^{[22]}$ Instead, the intrinsic radiative efficiency $\eta_{\mathrm{r}}$ mainly influences the amplitude, i.e., the maximum and minimum of the normalized decay rate. In conclusion, this type of optical modeling should enable us to fit measured triplet decay rates and extract both the anisotropy factor $a$ and radiative efficiency $\eta_{\mathrm{r}}$.

\subsection{Measurement of the emitter lifetime and modeling of the orientation}

To measure the change in triplet lifetime with ETL thickness, we performed transient electroluminescence measurements. The devices were electrically excited with $50 \mu$ s long rectangular voltage pulses (rise/fall time $<10 \mathrm{~ns}$ ) and the decay in luminance after the end of the pulses was recorded. The current density was kept below $3 \mathrm{~mA} \mathrm{~cm}^{-2}$ to ensure comparable electrical behavior for all ETL thicknesses (cf. Figure 1c) and to avoid TTA. Figure 5 shows two typical transients for the red-emitting OLEDs with ETLs of different thicknesses. All devices showed a mono-exponential decay; non-exponential processes such as $\mathrm{TTA}^{[4]}$, tripletpolaron-annihilation $^{[5]}$, or delayed exciton generation ${ }^{[23]}$ are not observed. The triplet lifetime of the device was extracted by fitting a mono-exponential decay function. The measured decay rates of all $\operatorname{Ir}(\mathrm{MDQ})_{2}(\mathrm{acac})$ - and $\operatorname{Ir}(\mathrm{ppy})_{3}$-based samples are summarized in Figure 6 (open symbols).

The measured triplet decay rates versus ETL thickness data were then fitted with Eq. (5), performing a least-square optimization of $\Gamma_{\mathrm{r}}, \Gamma_{\mathrm{nr}}$, and $a$ (Figure 6, solid lines). For 


\section{Submitted to

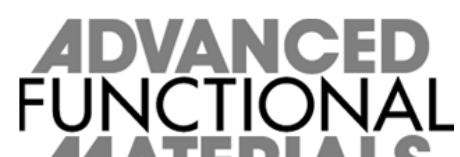 \\ MATERIALS}

$\operatorname{Ir}(\text { ppy })_{3}$-based OLEDs, the fit reveals an isotropic dipole orientation of $a=0.33 \pm 0.03$. By contrast, the transition dipoles of the $\operatorname{Ir}(\mathrm{MDQ})_{2}(\mathrm{acac})$ molecules in the red-emitting OLEDs clearly show a preferential horizontal orientation $(a=0.21 \pm 0.03)$; assuming an isotropic orientation for these devices leads to significant deviations between model and experiment for thin ETLs (cf. the dashed line in Figure 6a).

All fit parameters are summarized in Table 1. According to our fits, both emitters exhibit comparable intrinsic radiative efficiencies of $\eta_{\mathrm{r}} \approx 0.7$. Emitter orientation and radiative efficiency are consistent with the values obtained in previous measurements. ${ }^{[14-16,20,24,25]}$ However, in the past, time-resolved measurements of transition dipole orientations have been limited to optical excitation, ${ }^{[14,16]}$ under which location and width of the emission zone are different than under electrical excitation. Measuring orientation under electrical excitation more closely resembles the situation in the real device and ensures that one obtains the average orientation of exactly those emitter molecules that contribute to the electroluminescence generated by the device.

\subsection{Correlation of measured efficiency roll-off and emitter orientation}

Using the obtained fits, we are now able to properly describe and explain the measured critical current densities from Sec. 2.1. These $J_{0}$ values are scaled according to Eq. (4) to obtain the effective decay rates $\Gamma^{*}\left(\sqrt{J_{0}} \propto \Gamma^{*}\right)$ (Figure 6, solid symbols). A clear correlation between roll-off and triplet lifetime is observed for both emitters, i.e., the data extracted from $J_{0}$ is in good agreement with the fits obtained from transient electroluminescence measurements. Moreover, with knowledge of the emitter orientation (Sec. 2.3), the observed differences in roll-off behavior between the two emitters can be explained: Due to their 


\section{Submitted to

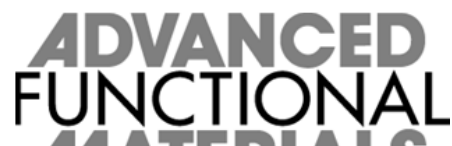 \\ MATERAAS}

isotropic orientation, the decay rate of $\operatorname{Ir}(\mathrm{ppy})_{3}$ molecules is considerably accelerated when these are in close proximity to the metal cathode. Therefore, the roll-off in $\operatorname{Ir}(\mathrm{ppy})_{3}$ based devices decreases as the distance between emitter and electrode becomes smaller, in agreement with previous observations. ${ }^{[18]}$ For $\operatorname{Ir}(\mathrm{MDQ})_{2}(\mathrm{acac})$, however, the preferential horizontal transition dipole orientation leads to a minimal roll-off at an ETL thickness in the range of $50-100 \mathrm{~nm}$.

The decay rates estimated from $J_{0}$ values mostly agree with the direct measurements within their experimental uncertainty. Remaining deviations are probably caused by the presence of other quenching mechanisms that we have not taken into account here but that may also influence the efficiency roll-off. Especially for thin ETLs, quenching is higher than expected. As shown in Figure 1c, at high voltage the current-voltage characteristics slightly differ for devices with different ETL thickness, which can lead to reduced charge balance at high voltages for certain devices.

\subsection{Calculation of the TTA rate constant $k_{\mathrm{TT}}$}

In the following, Eq. (4) is used to extract the TTA rate constant $k_{\mathrm{TT}}$ from the measured critical current densities and the effective decay rates. The width of the exciton formation zone is assumed to be $w=10 \mathrm{~nm}$ for both device structures as this value has been previously measured for similar OLED stacks based on EMLs of TCTA:Ir(ppy) $3 .{ }^{[5]}$ This yields $k_{\mathrm{TT}}=(1.9 \pm 0.2) \times 10^{-12} \mathrm{~cm}^{3} \mathrm{~s}^{-1}$ for $\operatorname{Ir}(\mathrm{MDQ})_{2}(\mathrm{acac})$ and $k_{\mathrm{TT}}=(6.0 \pm 1.2) \times 10^{-12} \mathrm{~cm}^{3} \mathrm{~s}^{-1}$ for $\operatorname{Ir}(\text { ppy })_{3}$. For the latter, a value of $k_{\mathrm{TT}}=(3 \pm 2) \times 10^{-12} \mathrm{~cm}^{3} \mathrm{~s}^{-1}$ has previously been obtained for a similar OLED stack using transient decay measurements at high excitation densities ${ }^{[5]}$. This agrees with our value within the range of errors. Compared to $\operatorname{Ir}(\mathrm{MDQ})_{2}(\mathrm{acac}), \operatorname{Ir}(\mathrm{ppy})_{3}$ shows a higher TTA rate, which is probably due to its stronger tendency to form 


\section{Submitted to

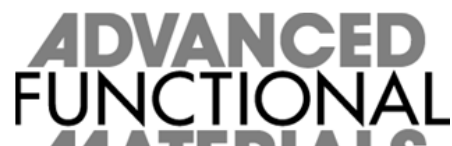

aggregates. ${ }^{[26]}$ We note that measurements of $k_{\mathrm{TT}}$ usually require time-resolved measurements at high excitation densities which can introduce device degradation. The method suggested here is instead based on a combination of decay time measurements at low excitation densities with measurements of the efficiency roll-off.

\subsection{Simulation of efficiency roll-off}

Optical simulations are frequently used to optimize light extraction (outcoupling) from OLEDs, but up to now they do not take efficiency roll-off into account. Instead, the thickness of all layers in the device is usually only optimized for low brightness levels. However, the optimum thickness may change at high brightness because the decay rate and thus the fraction of excitons lost to TTA generally depend on layer thickness. In the following, we combine optical simulations of EQE and decay rate (both as a function of ETL thickness) with calculations of the efficiency roll-off, using the $\operatorname{Ir}(\mathrm{MDQ})_{2}(\mathrm{acac})$ based OLEDs (Stack A) as an example.

According to Figure $2 \mathrm{a}$ and to simulations performed in Ref. ${ }^{[20]}$, Stack A reaches the highest EQE at low brightness for second maximum devices. Whether the second or the first maximum is more suitable, depends on the radiative efficiency and the orientation of the emitter molecules, as well as on other factors including the electroluminescence spectrum and the reflectivity of the cathode. As shown in Sec. 2.1, the efficiency roll-off depends on the position of the emitter within the OLED microcavity because different positions lead to different Purcell factors and thus different decay rates. Therefore, a first maximum OLED shows a different roll-off behavior than a second maximum device.

Figure 7a shows the ratio of the calculated EQE of the first maximum devices over the $\mathrm{EQE}$ of the second maximum device as a function of current density. The simulation is 


\section{Submitted to

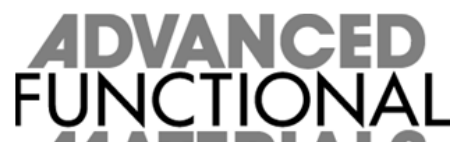 \\ MATERIALS}

performed for hypothetical phosphorescent emitters with different anisotropy factors $(0 \leq a \leq 0.33)$ and radiative efficiencies $\left(0.4 \leq \eta_{\mathrm{r}} \leq 1\right)$. The EQE roll-off is calculated according to Eq. (3) using the values of $k_{\mathrm{TT}}$ and $w$ as determined in Sec. 2.5. For the actual material parameters of $\operatorname{Ir}(\mathrm{MDQ})_{2}(\mathrm{acac})\left(a=0.21, \eta_{\mathrm{r}}=0.71\right)$, the second maximum device is more efficient up to a current density of $5 \mathrm{~mA} \mathrm{~cm}^{-2}$, which corresponds to a brightness of approximately $1300 \mathrm{~cd} \mathrm{~m}^{-2}$. At higher brightness levels, the first maximum device performs better. Furthermore, Figure 7a illustrates the influence of the anisotropy factor and the radiative efficiency: The EQE of the first maximum device would increase if the emitters were oriented more horizontally because losses from coupling of vertically oriented dipoles to the metal electrode are reduced. For emitters with high radiative efficiency, however, second maximum devices would become more efficient. In this case, the second maximum device can be the most efficient even for rather high brightness levels (e.g., up to $56 \mathrm{~mA} \mathrm{~cm}^{-2}$ for an emitter with $\left.a=0.33, \eta_{\mathrm{r}}=1\right)$.

To systematically evaluate how the emitter orientation and radiative efficiency influence the roll-off, we calculated the critical current density $J_{0}$ for the first maximum devices of Stack A as a function of emitter orientation and radiative efficiency (Figure $7 \mathrm{~b}$ ). For a given emitter orientation, the critical current density is highest for the highest radiative efficiency. This is related to the increase in decay rate at small ETL thickness (Figure 4b): If the radiative efficiency is high, the total decay rate of the emitter is dominated by the effective radiative decay rate, which in turn strongly increases in close proximity to the metal contact, thus reducing the roll-off. The influence of orientation, however, is less obvious: $J_{0}$ remains relatively constant for different values of $a$ if $\eta_{\mathrm{r}}$ is low, while for $\eta_{\mathrm{r}}=1$ a clear maximum of $J_{0}$ is obtained at an intermediate value of $a \approx 0.22$. The different behavior for low and high $\eta_{\mathrm{r}}$ is caused by the strong shift in the ETL thickness required to position the emitter molecules at 


\section{Submitted to

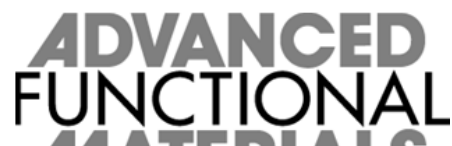 \\ MATERIALS}

the first maximum of the field. For instance, when changing $a$ from 0 to 0.33 (at $\eta_{\mathrm{r}}=1$ ), the ETL thickness changes by $18 \mathrm{~nm}$. Considering the properties of the actual $\operatorname{Ir}(\mathrm{MDQ})_{2}(\mathrm{acac})$, the average orientation of the transition dipoles in the material is close to the optimum with regard to the roll-off. However, the roll-off performance could be improved further if an emitter with higher radiative efficiency became available, which would of course also increase the absolute EQE.

A similar behavior was also found for Stack B containing $\operatorname{Ir}(\text { ppy })_{3}$ as emitter. However, at this stage, the dependence of EQE and roll-off on $a$ and $\eta_{\mathrm{r}}$ cannot be generalized because especially the reflectivity of the metal electrode has a pronounced influence on the EQE and the decay rates. Efficient device design therefore always requires optical simulation of the particular OLED stack.

\section{Conclusions}

In conclusion, we have shown that the efficiency roll-off is strongly influenced by the position and orientation of the emitter molecules within the OLED microcavity. The relation between efficiency roll-off and distance between emitter molecules and metal cathode was investigated in detail for OLEDs based either on $\operatorname{Ir}(\mathrm{MDQ})_{2}(\mathrm{acac})$ or on $\operatorname{Ir}(\mathrm{ppy})_{3}$. A distinctly different behavior was observed for the two types of emitter molecules. By performing timeresolved electroluminescence measurements and detailed optical modeling on the same set of OLEDs, we were able to correlate these differences to the different orientation of the emissive dipoles for the two types of emitters. OLEDs based on $\operatorname{Ir}(\mathrm{ppy})_{3}$, which is oriented isotropically, show the lowest roll-off if the emitter is positioned close to the metal cathode. $\operatorname{Ir}(\mathrm{MDQ})_{2}(\mathrm{acac})$, instead, is preferentially horizontally oriented. As a result, lowest roll-off is 


\section{Submitted to

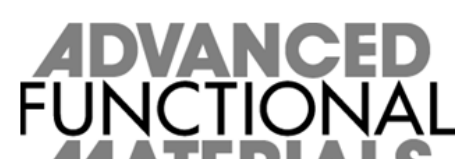

observed when the emitter is located close to the first optical maximum of the electromagnetic field. Due to the clear relation between roll-off and emitter orientation, the roll-off analysis introduced here can in principle be used to perform an independent in-situ measurement of emitter orientation. However, this requires that the contribution of roll-off mechanisms other than TTA can be neglected.

Our findings are important to guide the optimization of OLEDs towards even higher efficiency at high brightness: First, the good agreement between the decay rates estimated from measured critical current densities and the decay rates measured directly indicates that TTA is indeed the dominant mechanism for efficiency roll-off in our OLEDs. Other possible roll-off mechanisms show a different dependence on decay rate and, hence, their presence would cause strong deviations between the two sets of decay rates. Second, the distance of the emitter molecules from the metal contact of the OLED should be selected according to the emitter orientation, the radiative efficiency, and the current regime in which the OLED is operated. For OLEDs based on horizontally oriented emitters with lower radiative efficiency, the first optical maximum is typically more efficient for all brightness levels. Stacks based on isotropic emitters with high radiative efficiency show higher EQE for second maximum devices at low currents, where no roll-off is observed. When going to high-brightness applications, however, most emitters perform best in first maximum devices, as the decay rate for these is often higher than in second maximum devices.

\section{Experimental Section}

Device fabrication: Organic materials are evaporated in a UHV-chamber (Kurt J. Lesker Co.) at a base pressure of $10^{-8}$ mbar onto glass substrates containing a $90 \mathrm{~nm}$ thick pre-coated indium tin oxide anode. The ETL thickness is varied using shadow masks allowing 


\section{Submitted to

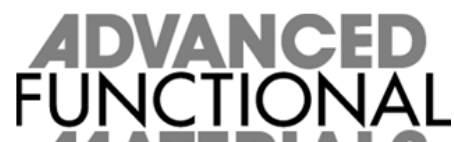 \\ MATERIALS}

fabrication of all samples within one run to ensure high comparability. The thickness is measured in-situ using quartz crystal monitors. All samples are encapsulated under nitrogen atmosphere using glass lids and epoxy resin directly after fabrication. Each sample contains four OLEDs with an active area of $6.62 \mathrm{~mm}^{2}$ each.

Materials: The OLEDs consist of an 2,2',7,7'-tetrakis( $N, N^{\prime}$-di- $p$ - methylphenylamino)9,9'-spirobifluorene (Spiro-TTB) hole transport layer, doped with 4 wt.\% 2,2'(perfluoronaphthalene-2,6-diylidene)dimalononitrile (F6-TCNNQ), a 2,2',7,7'-tetrakis- ( $N, N$ diphenylamino)-9,9'-spirobifluoren (Spiro-TAD) electron blocking layer, a bis-(2-methyl-8chinolinolato)-(4-phenyl-phenolato)-aluminium(III) (BAlq 2 ) hole blocking layer, a Cs-doped 4,7-diphenyl-1,10- phenanthroline (BPhen) ETL, and an Ag cathode. The EML consists of an N,N'-di(naphtalene-1-yl)-N,N'-diphenylbenzidine (NPB) matrix doped with $10 \mathrm{wt} . \%$ iridium(III)bis(2-methyldibenzo[f,h]quinoxaline) (acetylacetonate) $\left(\operatorname{Ir}(\mathrm{MDQ})_{2}(\mathrm{acac})\right)$ (Stack A) or a double emission layer of hole-transporting 4,4',4"'-tris(N-carbazolyl)triphenylamine (TCTA) and electron-transporting 2,2',2' '-(1,3,5-benzinetriyl)tris(1-phenyl-1$H$-benzimidazole) (TPBi), each doped with $8 \mathrm{wt.} \%$ fac-tris(2-phenylpyridine) iridium $\left(\operatorname{Ir}(\mathrm{ppy})_{3}\right)($ Stack B). All materials were purchased from commercial suppliers and purified further by vacuum gradient sublimation prior to use.

Sample characterization: Current density-voltage-luminance characteristics and spectral radiance of all samples are measured with an automated system containing a source-measure unit (Keithley SM2400), a calibrated spectrometer (Instrument Systems GmbH CAS140CT), and a silicon photodiode. The EQE is calculated after measuring angular dependent spectra using a goniometer, which contains a calibrated spectrometer (Ocean Optics USB4000). Time-resolved triplet lifetime measurements of the OLEDs are performed under electrical excitation (applying $50 \mu$ s long voltage pulses with a pulse generator, Hewlett Packard 
Submitted to

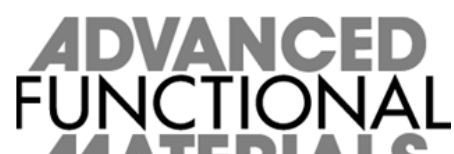

MATERIALS

8114A) using a streak camera (Hamamatsu C5680). The current density is detected simultaneously via an oscilloscope (Hewlett Packard Infinium 54815A).

\section{Acknowledgements}

This work was financed in part by the European Social Fund and the free state of Saxony through the OrganoMechanics project. This work is partly supported by the German Research Foundation (DFG) within the Cluster of Excellence 'Center for Advancing Electronics Dresden'. M.C.G. acknowledges support from the Daimler and Benz Foundation.

Received: ((will be filled in by the editorial staff))

Revised: ((will be filled in by the editorial staff)) Published online: ((will be filled in by the editorial staff))

[1] M. A. Baldo, D. F. O’Brien, Y. You, A. Shoustikov, S. Sibley, M. E. Thompson, S. R. Forrest, Nature 1998, 395, 151.

[2] S. Reineke, F. Lindner, G. Schwartz, N. Seidler, K. Walzer, B. Lüssem, K. Leo, Nature 2009, 459, 234.

[3] Z. B. Wang, M. G. Helander, J. Qiu, D. P. Puzzo, M. T. Greiner, Z. M. Hudson, S. Wang, Z. W. Liu, Z. H. Lu, Nature Photonics 2011, 5, 753.

[4] M. A. Baldo, C. Adachi, S. R. Forrest, Physical Review B 2000, 62, 10967.

[5] S. Reineke, K. Walzer, K. Leo, Physical Review B 2007, 75, 125328.

[6] N. Giebink, S. R. Forrest, Physical Review B 2008, 77, 235215.

[7] C. Murawski, K. Leo, M. C. Gather, Efficiency Roll-Off in Organic Light-Emitting Diodes. Advanced Materials 2013, accepted.

[8] N. C. Erickson, R. J. Holmes, Advanced Functional Materials 2013, DOI: 10.1002/adfm.201300101. 
[9] M. A. Baldo, M. E. Thompson, S. R. Forrest, Pure and Applied Chemistry 1999, 71, 2095.

[10] M. A. Baldo, S. Lamansky, P. E. Burrows, M. E. Thompson, S. R. Forrest, Applied Physics Letters 1999, 75, 4.

[11] T. Tsutsui, M. Yang, M. Yahiro, K. Nakamura, T. Watanabe, T. Tsuji, Y. Fukuda, T. Wakimoto, S. Miyaguchi, Japanese Journal of Applied Physics 1999, 38, 1502.

[12] L. Han, D. Yang, W. Li, B. Chu, Y. Chen, Z. Su, D. Zhang, F. Yan, Z. Hu, Z. Zhang, Applied Physics Letters 2008, 93, 153303.

[13] K. H. Drexhage, Journal of Luminescence 1970, 1-2, 693.

[14] T. D. Schmidt, D. S. Setz, M. Flämmich, J. Frischeisen, D. Michaelis, B. C. Krummacher, N. Danz, W. Brütting, Applied Physics Letters 2011, 99, 163302.

[15] S. Mladenovski, S. Hofmann, S. Reineke, L. Penninck, T. Verschueren, K. Neyts, Journal of Applied Physics 2011, 109, 083114.

[16] L. Penninck, F. Steinbacher, R. Krause, K. Neyts, Organic Electronics 2012, 13, 3079.

[17] Q. Huang, S. Reineke, K. Walzer, M. Pfeiffer, K. Leo, Applied Physics Letters 2006, $89,263512$.

[18] D. Song, S. Zhao, H. Aziz, Advanced Functional Materials 2011, 21, 2311.

[19] K. Walzer, B. Maennig, M. Pfeiffer, K. Leo, Chemical Reviews 2007, 107, 1233.

[20] M. Furno, R. Meerheim, S. Hofmann, B. Lüssem, K. Leo, Physical Review B 2012, 85, 115205.

[21] S. Reineke, G. Schwartz, K. Walzer, K. Leo, Applied Physics Letters 2007, 91, 123508.

[22] P. T. Worthing, R. M. Amos, W. L. Barnes, Physical Review A 1999, 59, 865.

[23] C. Weichsel, L. Burtone, S. Reineke, S. I. Hintschich, M. C. Gather, K. Leo, B. Lüssem, Physical Review B 2012, 86, 075204.

[24] M. Flämmich, J. Frischeisen, D. S. Setz, D. Michaelis, B. C. Krummacher, T. D. Schmidt, W. Brütting, N. Danz, Organic Electronics 2011, 12, 1663.

[25] P. Liehm, C. Murawski, M. Furno, B. Lüssem, K. Leo, M. C. Gather, Applied Physics Letters 2012, 101, 253304.

[26] S. Reineke, T. C. Rosenow, B. Lüssem, K. Leo, Advanced Materials 2010, 22, 3189. 


\section{Submitted to}

\section{FUNCTIONAL \\ MATERIALS}

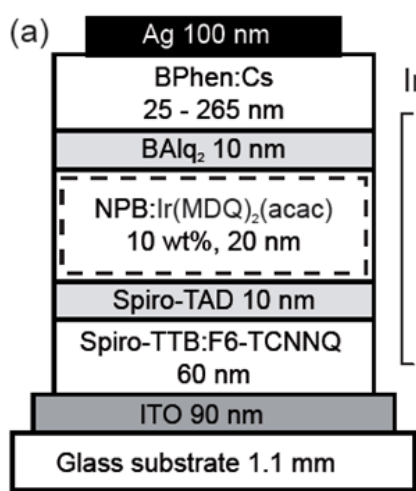

Stack A
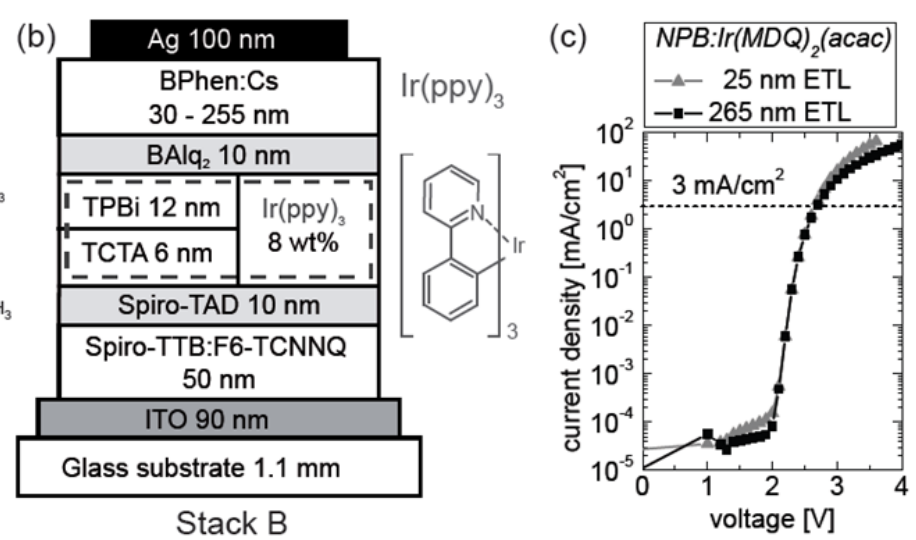

Figure 1. Structure of (a) the red-emitting $\operatorname{Ir}(\mathrm{MDQ})_{2}(\mathrm{acac})$ based OLEDs (Stack A) and (b) the green-emitting $\operatorname{Ir}(\mathrm{ppy})_{3}$ based OLEDs (Stack B) investigated in this study. Molecular structures of the phosphorescent emitter molecules $\operatorname{Ir}(\mathrm{MDQ})_{2}(\mathrm{acac})$ and $\operatorname{Ir}(\mathrm{ppy})_{3}$ are also shown. The thickness of the BPhen:Cs electron transport layer (ETL) was varied over a broad range as indicated. (c) Current density-voltage characteristics of the two $\operatorname{Ir}(\mathrm{MDQ})_{2}(\mathrm{acac})$ based OLEDs with thinnest/thickest BPhen:Cs layer. 


\section{Submitted to}

(a)

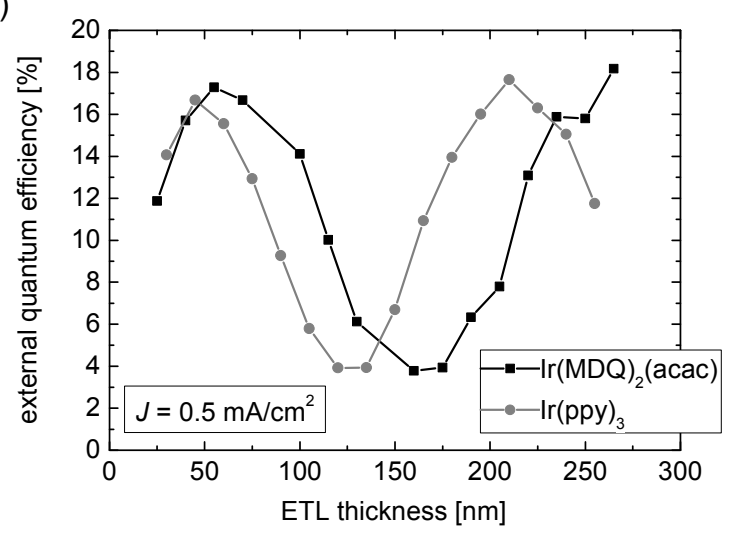

(b)

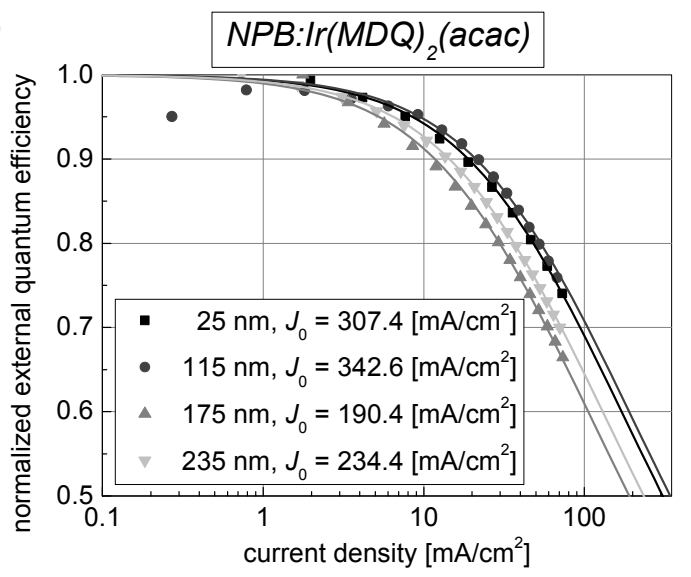

Figure 2. (a) External quantum efficiency as a function of ETL thickness for the red and green emitting OLEDs shown in Figure 1 at an applied current density of $0.5 \mathrm{~mA} \mathrm{~cm}^{-2}$. Lines are guides to the eye. (b) Normalized EQE versus current density for several red-emitting OLEDs with different ETL thickness. Experimental data (points) and fits according to Eq. (3) (lines). 
(a)

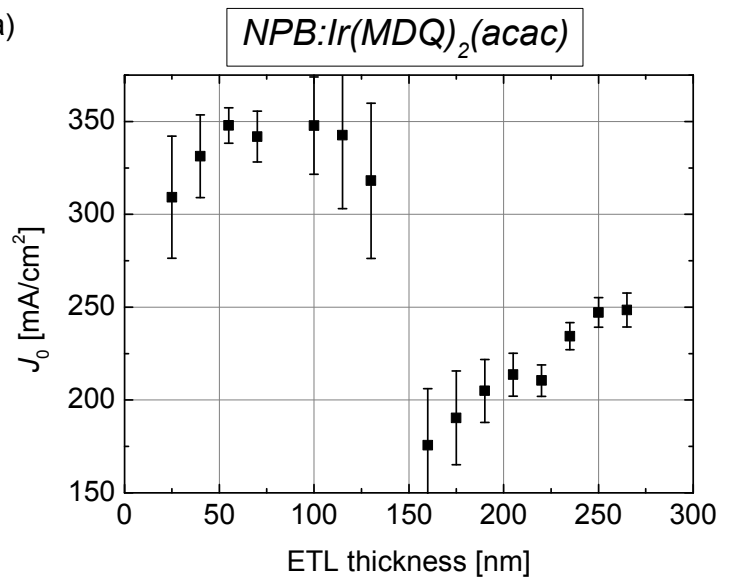

(b)

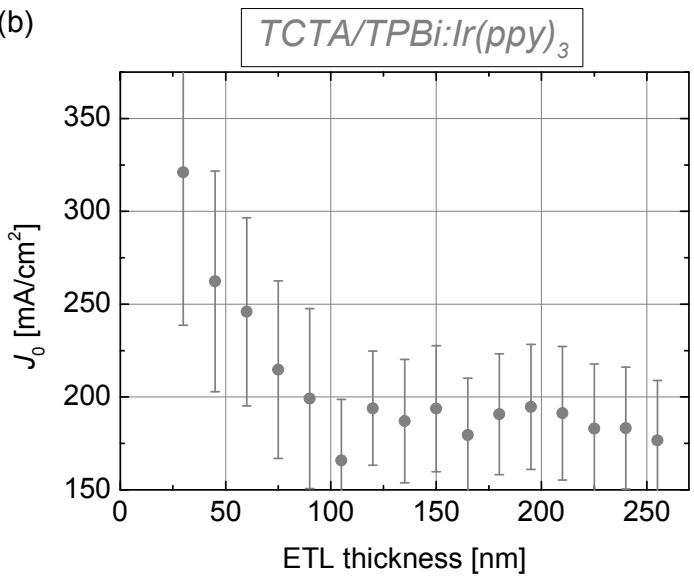

Figure 3. Critical current density $J_{0}$ as a function of ETL thickness, obtained from fits of the EQE vs. current density using Eq. (3). Data is shown for OLEDs based on (a) $\operatorname{Ir}(\mathrm{MDQ})_{2}$ (acac) and (b) $\operatorname{Ir}(\text { ppy })_{3}$. Error bars represent uncertainty of fit to EQE vs. current density data.

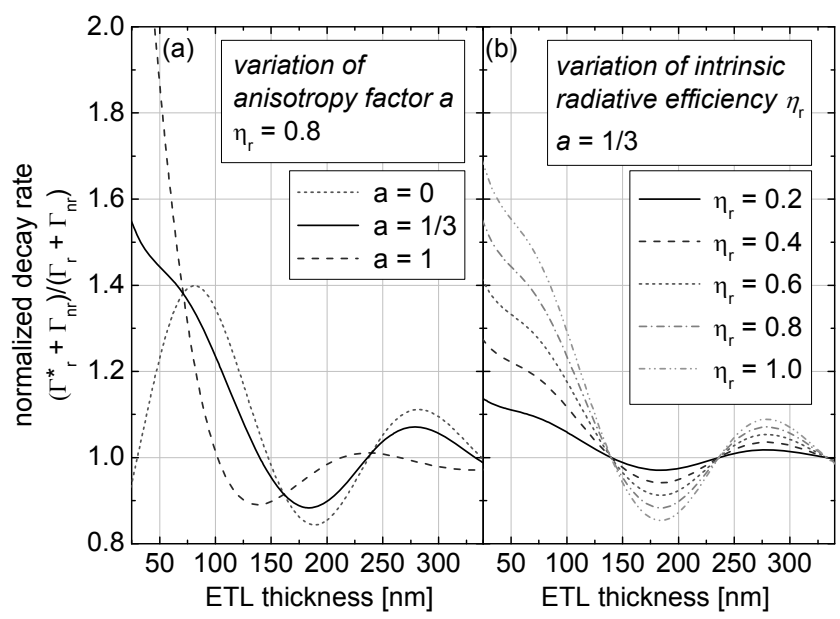

Figure 4. Normalized triplet decay rate as a function of the ETL thickness of $\operatorname{Ir}(\mathrm{MDQ})_{2}(\mathrm{acac})$ based OLEDs (Stack A). Data obtained using optical modeling. (a) For different average emissive dipole orientations $a$ of the $\operatorname{Ir}(\mathrm{MDQ})_{2}(\mathrm{acac})$ molecules in the device (assuming $\left.\eta_{\mathrm{r}}=0.8\right)$, where $a=(0,1 / 3,1)$ stands for (horizontal, isotropic, vertical) orientation. (b) For different intrinsic radiative efficiencies $\eta_{\mathrm{r}}$ of the $\operatorname{Ir}(\mathrm{MDQ})_{2}(\mathrm{acac})$ molecules (assuming $a=1 / 3)$. 


\section{Submitted to \\ FUNCTIONAL \\ MATERIALS}

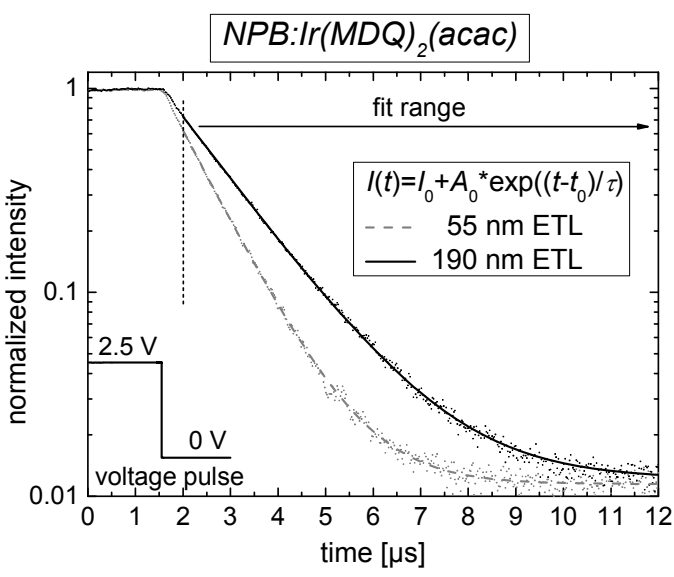

Figure 5. Transient electroluminescent intensity data for two typical samples based on $\operatorname{Ir}(\mathrm{MDQ})_{2}$ (acac) after excitation with a $50 \mu$ s long voltage pulse of $2.5 \mathrm{~V}$ (points). The data is fitted with a mono-exponential decay function to extract the triplet lifetime $\tau^{*}$ (lines). 


\section{Submitted to \\ ADNANCER
FUNCIONALL}

(a)

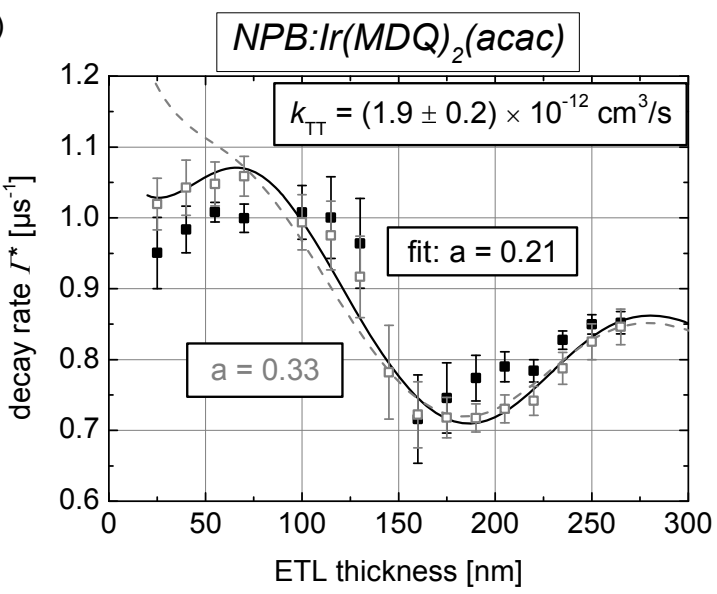

(b)

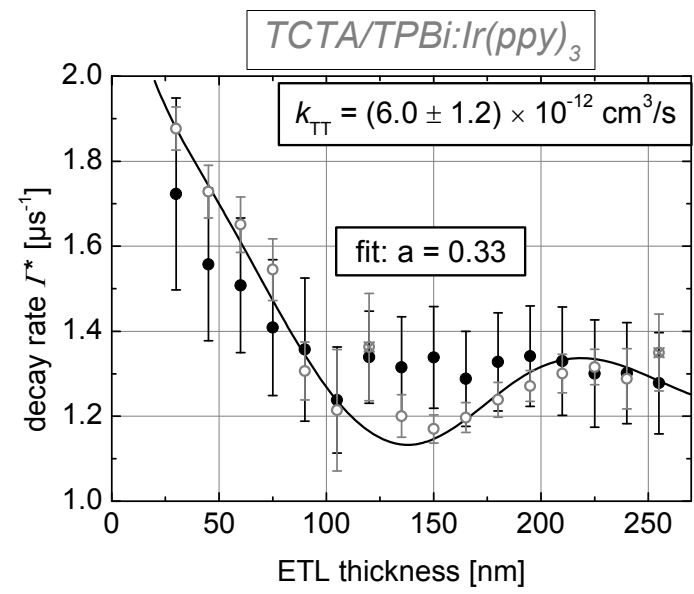

Figure 6. Directly measured decay rates $\Gamma^{*}$ (open symbols, error bars represent uncertainty of fit to time-resolved intensity data) and decay rates extracted from fitted $J_{0}$ values (closed symbols, errors bars according to Fig. 3) for OLEDs based on (a) $\operatorname{Ir}(\mathrm{MDQ})_{2}(\mathrm{acac})$ and (b) $\operatorname{Ir}(\text { ppy })_{3}$. The directly measured decay rates are fitted according the model described in the text (Eq. 5; solid lines; crossed symbols represent data that was excluded from the fitting routine). For $\operatorname{Ir}(\mathrm{MDQ})_{2}(\mathrm{acac})$ an anisotropy factor of $a=0.21$ is obtained while $\operatorname{Ir}(\mathrm{ppy})_{3}$ dipoles are isotropically oriented $(a=0.33)$. For comparison, the dashed line in (a) shows the expected behavior if the emissive dipoles of $\operatorname{Ir}(\mathrm{MDQ})_{2}(\mathrm{acac})$ were oriented isotropically. 

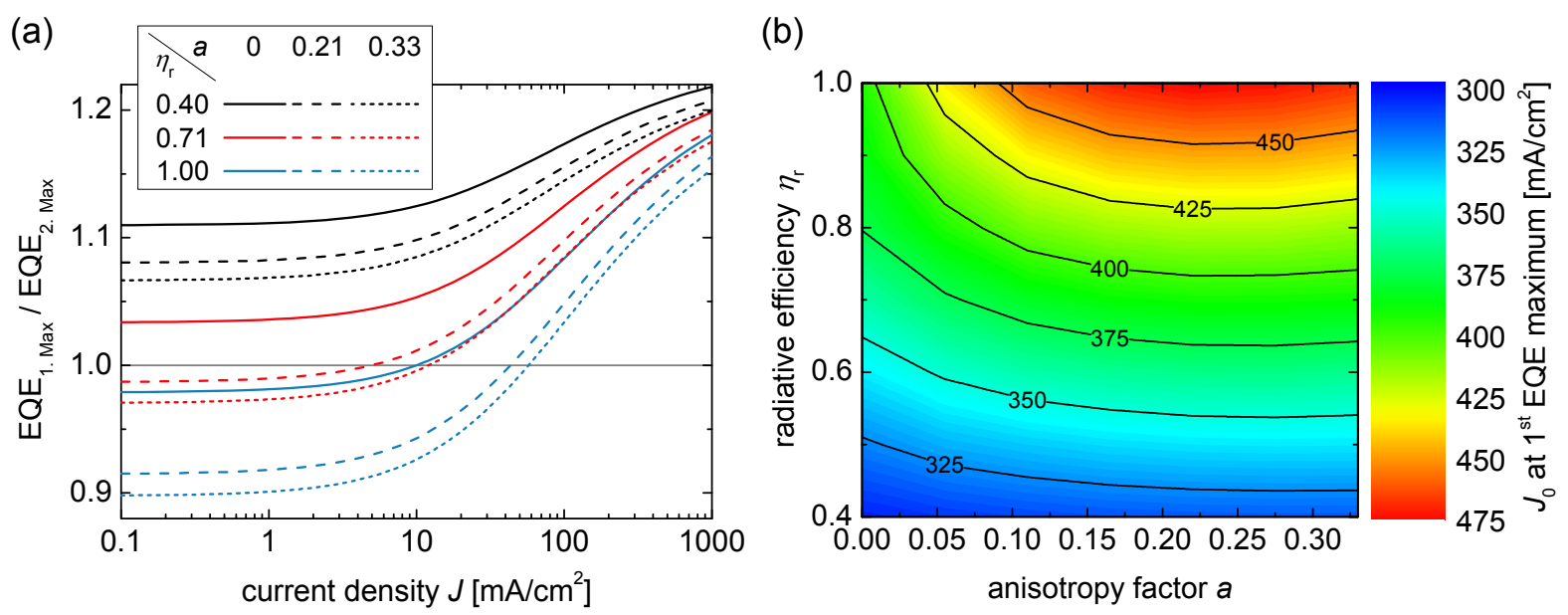

Figure 7. Optical simulation of efficiency roll-off for OLEDs based on Stack A for varying anisotropy factor $a$ and radiative efficiency $\eta_{\mathrm{r}}$ assuming a constant intrinsic decay rate of $\Gamma=0.816 \mu \mathrm{s}^{-1}$ and the emission spectrum of $\operatorname{Ir}(\mathrm{MDQ})_{2}$ (acac). (a) Ratio of EQE for first maximum devices over EQE of second maximum devices as function of the current density $J$. Values above 1 indicate that the first maximum device is more efficient. (b) Critical current density $J_{0}$ of devices with emitters located in first EQE maximum (z-scale) as a function of $\eta_{\mathrm{r}}$ and $a$.

Table 1. Extracted fit parameters for OLEDs containing $\operatorname{Ir}(\mathrm{MDQ})_{2}(\mathrm{acac})$ or $\operatorname{Ir}(\mathrm{ppy})_{3}$ : anisotropy factor $a$, radiative decay rate $\Gamma_{\mathrm{r}}$, non-radiative decay rate $\Gamma_{\mathrm{nr}}$, radiative efficiency $\eta_{\mathrm{r}}$, and TTA rate constant $k_{\mathrm{TT}}$.

\begin{tabular}{lccccc}
\hline & $a$ & $\Gamma_{\mathrm{r}}\left[\mu \mathrm{s}^{-1}\right]$ & $\Gamma_{\mathrm{nr}}\left[\mu \mathrm{s}^{-1}\right]$ & $\eta_{\mathrm{r}}$ & $k_{\mathrm{TT}}\left[\mathrm{cm}^{3} \mathrm{~s}^{-1}\right]$ \\
\hline $\operatorname{Ir}(\mathrm{MDQ})_{2}(\mathrm{acac})$ & $0.21 \pm 0.03$ & $0.58 \pm 0.03$ & $0.24 \pm 0.03$ & $0.71 \pm 0.06$ & $(1.9 \pm 0.2) \times 10^{-12}$ \\
$\operatorname{Ir}(\mathrm{ppy})_{3}$ & $0.33 \pm 0.03$ & $0.92 \pm 0.04$ & $0.36 \pm 0.04$ & $0.72 \pm 0.03$ & $(6.0 \pm 1.2) \times 10^{-12}$ \\
\hline
\end{tabular}




\section{Submitted to

\section{Table of contents entry}

Keyword: OLED, efficiency roll-off, emitter orientation, decay rate, triplet-triplet-annihilation

Caroline Murawski, Philipp Liehm, Karl Leo, and Malte C. Gather:

Influence of cavity thickness and emitter orientation on the efficiency roll-off of phosphorescent organic light-emitting diodes

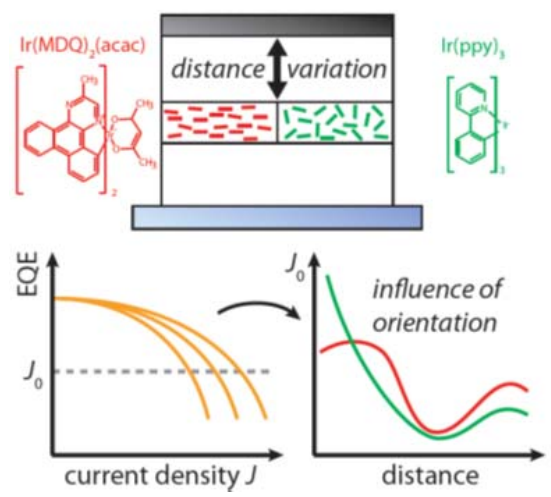

The efficiency roll-off of organic light-emitting diodes is studied by varying the distance between emitter and metal cathode. Roll-off is found to depend on the orientation of the emitter molecules, which can be explained by measurements and modeling of the triplet decay rate. Simulations of the roll-off behavior give further insight into the principles of efficient stack design. 